\title{
Synthesis of $\boldsymbol{\alpha}, \boldsymbol{\beta}$-diamino acid derivatives via asymmetric Mannich reaction of glycine imino esters catalyzed by a chiral phosphoramidite· silver complex
}

\author{
Alberto Cayuelas, ${ }^{\mathrm{a}}$ Loane Serrano, ${ }^{\mathrm{a}}$ Carmen Nájera, ${ }^{\mathrm{a}}$ and José M. Sansano, ${ }^{\mathrm{a} *}$ \\ a Departamento de Química Orgánica, Instituto de Síntesis Orgánica (ISO), and Centro de Innovación en \\ Química Avanzada (ORFEO-CINQA). Universidad de Alicante, Apdo. 99, E-03080-Alicante, Spain. \\ Fax: (+34)-965-903-549; phone: (+34)-965-903-549; e-mail: cnajera@ua.es, jmsansano@ua.es
}

Dedicated to the memory of Prof. Ekkehard Winterfeldt

Abstract: AgOTf-Phosphoramidite complexes catalyze efficiently the enantioselective Mannichtype reaction between benzophenone-imine glycine methyl ester and $N$-tosyl aldimines in the absence of base. The corresponding syn-adducts, which are direct precursors of $\alpha, \beta$-diamino acids, are obtained in moderate to good syn-diastereoselectivities (up to 9:1) and high enantioselectivities (up to $99 \%$ ee).

\section{Introduction}

The asymmetric Mannich reaction is a very useful methodology for the preparation of enantioenriched $\beta$-amino carbonyl compounds where the absolute configuration of two stereogenic centers can be defined simultaneously. ${ }^{1,2,3}$ There are many type of enolates which are able to add to imines by intermediacy of chiral metal complexes or organocatalysts. Interestingly, in the case of using Schiff bases derived from $\alpha$-amino esters 1 as nucleophiles and imines 2, protected $\alpha, \beta$ diamino esters $\mathbf{3}$ can be straightforwardly prepared. After deprotection $\alpha, \beta$-diamino acids $\mathbf{4}$ are easily generated, which are biologically relevant ${ }^{4}$ and also chemically attractive due to their use as chiral building blocks ${ }^{5}$ (Scheme 1). Apart from this Mannich-type reaction, the asymmetric amination of $\alpha, \beta$-unsaturated acid derivatives, and the catalytic hydrogenation of bis(amido)acrylates diamino are two additional routes to achieve them. ${ }^{6}$

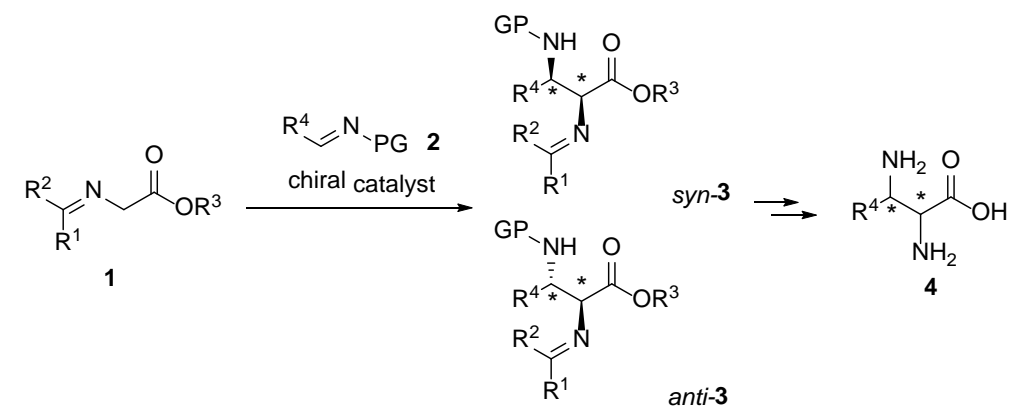

Scheme 1. General Mannich-type reaction between imino esters and $N$-protected imines 
The enantioselective reaction of imino esters 1 with $N$-protected imines shown in Scheme 1 has been successfully performed in the presence of chiral organocatalysts $5^{7 \mathrm{a}}$ and $67^{\mathrm{b}}$ or chiral metal complexes $\mathbf{7 - 1 5 ^ { 8 }}$ (Figure 1). The reactions involving organocatalysts 5 and $\mathbf{6}$ afforded almost exclusively the syn-3 diastereoisomer with excellent enantioselections. ${ }^{9}$ The syn-diasteroisomer was also the major isolated product when chiral copper complexes were employed. The unique exception was registered when the major anti-3 diastereoisomer was isolated in good enantioselectivities after the reaction with copper(I) complexes 11 and 12. In the case of aldimino esters $1\left(\mathrm{R}^{1}=\mathrm{H}, \mathrm{R}^{2}=\mathrm{Ph}\right)$ the anti-3 diastereoisomers were isolated with excellent enantioselectivities, a bulky $N$-(8-quinolyl)sulfonyl protecting group being needed. $8^{\mathrm{i}}$ Copper salts are mainly employed and only one contribution employed a chiral 15· silver acetate complex affording the syn-diasteroisomers as major products. $8^{\mathrm{k}}$

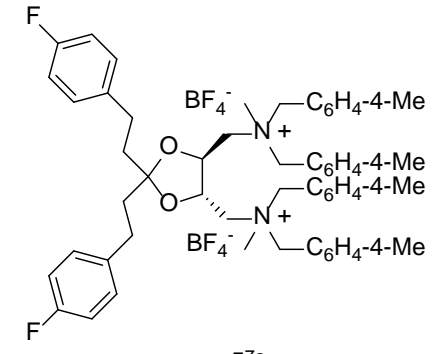

$5^{7 a}$

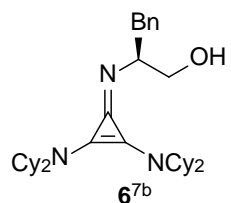

$6^{7 \mathrm{~b}}$
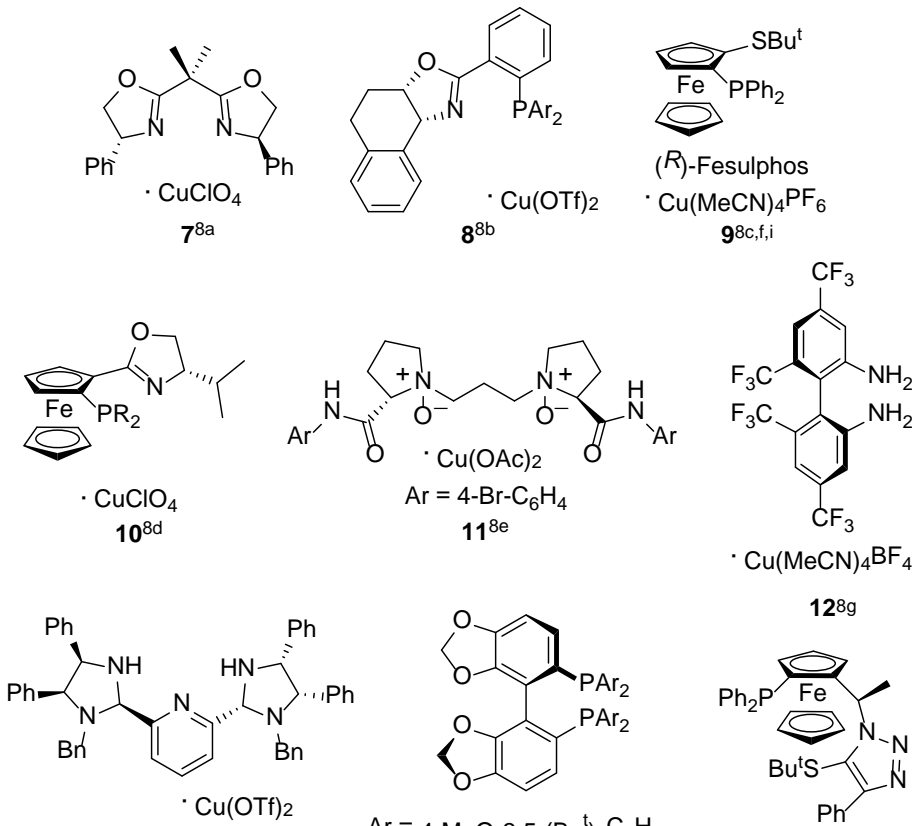

$13^{8 \mathrm{~h}}$

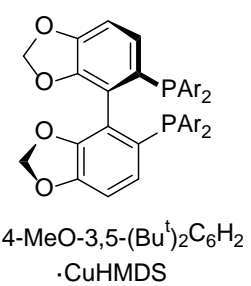

$148 \mathrm{j}$

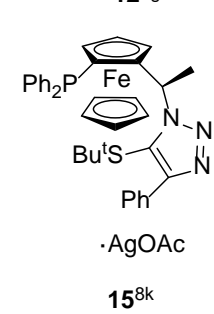

Figure 1. Chiral catalysts employed in the Mannich-type reaction between imino esters and $\mathrm{N}$ protected imines. 
We have recently described that complexes formed by silver salts and privileged chiral ligands ${ }^{10}$ such as Binap $\mathbf{1 6}^{11}$ and phosphoramidites $\mathbf{1 7}$ and $\mathbf{1 8}^{12}$ are excellent catalysts for enantioselective 1,3-dipolar cycloadditions ${ }^{13,14}$ of azomethine ylides derived from imino esters $\mathbf{1}$ in the construction of enantiomerically enriched highly substituted prolines. ${ }^{15,16}$ In this work, we will describe the application of these silver chiral complexes ${ }^{17}$ in the Mannich type reaction of glycine benzophenone Schiff bases and protected aldimines.

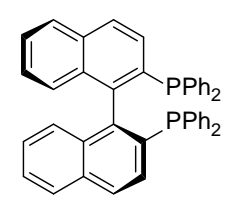

(Sa)-Binap 16

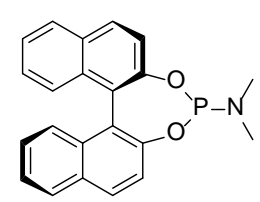

( $R_{\mathrm{a})}$-Monophos 17

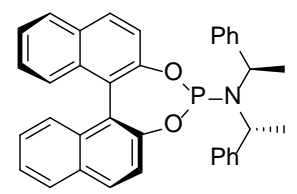

$\left(S_{\mathrm{a}}, R, R\right)-18$

Figure 2. Chiral privileged ligands 16-18 employed in this study.

\section{Results and Discussion}

The selected reaction for the optimization conditions involved the glycine benzophenone imine derivative 1a $\left(\mathrm{R}^{1}=\mathrm{R}^{2}=\mathrm{Ph}, \mathrm{R}^{3}=\mathrm{H}\right.$ and $\mathrm{R}^{4}=\mathrm{Me}$ ), benzaldehyde $N$-tosylimine $\mathbf{2 a}(\mathrm{PG}=\mathrm{Ts}$ ), triethylamine (5 mol\%) as base, and AgOAc (5 mol\%) in toluene as solvent at room temperature for $1 \mathrm{~d}$ (Scheme 2). The reaction performed with (S)-Binap 16 was first evaluated in the presence and in the absence of triethylamine. High conversions and 67:33 syn:anti diastereomeric ratios of compound 3a were obtained in both cases. Surprisingly, the highest enantioselection was observed for the syn-diastereoisomer when no base was added (Table 1, entries 1 and 2). The same behavior was exhibited by the chiral catalyst formed by Monophos 17 and silver acetate. A higher 75:25 $d r$ was observed but with lower enantioselections (Table 1, entries 3 and 4). Phosphoramidite 18 and AgOAc offered a higher enantioselectivity (80\% ee) and 80:20 dr when the reaction was carried out in the absence of base in (Table 1, compare entries 5 and 6). Copper(I) and copper(II) triflates were also surveyed (not shown in Table 1 ) giving a complex crude reaction mixture ( ${ }^{1} \mathrm{H}$ NMR) from no completed reactions. The reactions run under conditions shown in entries 5 and 6 became very slow at $0{ }^{\circ} \mathrm{C}$.

Silver perchlorate was a suitable metal salt for phosphoramidite $\mathbf{1 8}$ affording major synstereoisomer 3a in high conversion but with lower diastereoselection (72:28) than the observed for the reaction run with AgOAc (Table 1, compare entries 5 with 6, and 6 with 8). In light of all these tests the reaction with different silver salts was next essayed without triethylamine. Thus, AgTfa 
(Tfa = trifluoroacetate), $\mathrm{AgOBz}, \mathrm{AgSbF}_{6}$, and $\mathrm{Ag}_{2} \mathrm{CO}_{3}$ did not improve the enantioselection generated by the employment of silver perchlorate (Table 1, entries 9-12). However, the best results were observed when AgOTf was employed as silver salt (Table 1, entry 13).

In order to ameliorate the diastereoselectivity of the process gaining some enantioselection, different nitrogen protecting groups for the imines such as nosyl ( $p$-nitrophenylsulfonyl, Ns) and $N$ (8-quinolyl)sulfonyl (8-Q) were evaluated. In both cases conversions were high but the enantioselection was very poor (Table 1, entries 14, and 15), even when the tert-butyl (diphenylmethylene)glycinate was used (Table 1, entries 16-18).

The influence of the solvent was also noticeable. Toluene was the best solvent whereas THF, $\mathrm{Et}_{2} \mathrm{O}$, DCM, and MeCN furnished both lower $d r$ and ee (Table 1, entries 19-22). The mechanism of this reaction would be very similar than the reported one for the asymmetric synthesis of polysubstituted prolines (in its first stage) through a 1,3-dipolar cycloaddition reaction using the same catalytic system. ${ }^{12 b}$

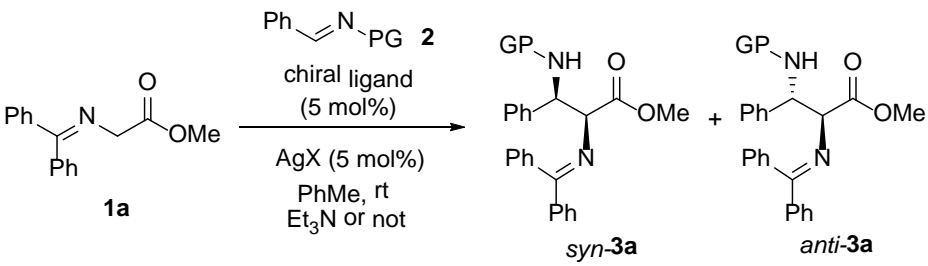

Scheme 2. Optimization reaction model.

Table 1. Optimization reactions.

\begin{tabular}{|c|c|c|c|c|c|c|c|}
\hline Ent. & AgX & Ligand & Base & PG & Yield (\%) ${ }^{\mathrm{a}}$ & $d r(\text { syn:anti) })^{a}$ & $e e(s y n, a n t i)^{b}$ \\
\hline 1 & $\mathrm{AgOAc}$ & 16 & TEA & Ts & 100 & $67: 33$ & 12,20 \\
\hline 2 & AgOAc & 16 & - & Ts & 100 & $67: 33$ & 24,2 \\
\hline 3 & $\mathrm{AgOAc}$ & 17 & TEA & Ts & 100 & $75: 25$ & $<10,<10$ \\
\hline 4 & AgOAc & 17 & - & Ts & 100 & $75: 25$ & 10,6 \\
\hline 5 & $\mathrm{AgOAc}$ & 18 & TEA & Ts & 90 & 80:20 & 12,24 \\
\hline 6 & $\mathrm{AgOAc}$ & 18 & - & Ts & 90 & 80:20 & 80,10 \\
\hline 7 & $\mathrm{AgClO}_{4}$ & 18 & TEA & Ts & 100 & $86: 14$ & 12 , rac. \\
\hline 8 & $\mathrm{AgClO}_{4}$ & 18 & - & Ts & 75 & $72: 28$ & 92,32 \\
\hline 9 & AgTfa & 18 & - & Ts & 85 & $60: 40$ & 14,12 \\
\hline 10 & $\mathrm{AgOBz}$ & 18 & - & Ts & 100 & $75: 25$ & 78,38 \\
\hline 11 & $\mathrm{AgSbF}_{6}$ & 18 & - & Ts & 85 & $60: 40$ & 64,16 \\
\hline 12 & $\mathrm{Ag}_{2} \mathrm{CO}_{3}$ & 18 & - & Ts & 92 & $84: 16$ & 8,4 \\
\hline 13 & AgOTf & 18 & - & Ts & 94 & $72: 28$ & 96,58 \\
\hline 14 & AgOTf & 18 & - & $\mathrm{Ns}^{\mathrm{c}}$ & 100 & $90: 10$ & 24,18 \\
\hline 15 & AgOTf & 18 & - & $8-Q^{d}$ & 90 & $72: 28$ & 6,2 \\
\hline
\end{tabular}




\begin{tabular}{llllllll}
16 & AgOTf $^{\mathrm{e}}$ & $\mathbf{1 8}$ & - & $\mathrm{Ts}$ & 95 & $80: 20$ & 4,28 \\
17 & AgOTf $^{\mathrm{e}}$ & $\mathbf{1 8}$ & - & $\mathrm{Ns}^{\mathrm{c}}$ & 100 & $>96: 4$ & 56, rac. \\
18 & AgOTf $^{\mathrm{e}}$ & $\mathbf{1 8}$ & - & $8-\mathrm{Q}^{\mathrm{d}}$ & 85 & $86: 14$ & 10,6 \\
19 & AgOTf $^{\mathrm{f}}$ & $\mathbf{1 8}$ & - & $\mathrm{Ts}$ & 89 & $67: 33$ & 38,6 \\
20 & AgOTf $^{\mathrm{g}}$ & $\mathbf{1 8}$ & - & $\mathrm{Ts}$ & 92 & $72: 28$ & rac., rac. \\
21 & AgOTf $^{\mathrm{h}}$ & $\mathbf{1 8}$ & - & $\mathrm{Ts}$ & 91 & $62: 38$ & 58,86 \\
22 & AgOTf $^{\mathrm{i}}$ & $\mathbf{1 8}$ & - & $\mathrm{Ts}$ & 87 & $72: 28$ & 24,40 \\
\hline
\end{tabular}

${ }^{\mathrm{a}}$ Determined by ${ }^{1} \mathrm{H} \mathrm{RMN}$ of the crude reaction mixture.

${ }^{\mathrm{b}}$ Determined by HPLC using chiral columns.

${ }^{\mathrm{c}} p$-Nitrophenylsulfonyl group (nosyl).

${ }^{\mathrm{d}} \mathrm{N}$-(8-quinolyl)sulfonyl group.

e The corresponding tert-butyl ester was employed.

${ }^{\mathrm{f}}$ Reaction run in the presence of THF as solvent.

${ }^{\mathrm{g}}$ Reaction run in the presence of $\mathrm{Et}_{2} \mathrm{O}$ as solvent.

${ }^{\mathrm{h}}$ Reaction run in the presence of DCM as solvent.

${ }^{\mathrm{i}}$ Reaction run in the presence of MeCN as solvent.

The scope of the reaction was investigated under the optimized reaction conditions using toluene as solvent at room temperature. The reaction catalyzed by the complex AgOTf $\left(S_{\mathrm{a}}, R, R\right)$-18 gave the same results than those reported by using $\left(R_{\mathrm{a}}, S, S\right)-\mathbf{1 8}$ ligand but obtaining the opposite enantiomeric form ent-3 (Table 2, entry 2). Different aldimines were prepared according to known methods and directly employed as electrophiles in the titled Mannich type reaction with imino ester 1a. In general, if a comparison is established between product 3a (Table 2, entry 1) and the different $\mathbf{3 b}$-3l molecules, the presence of a substituent in the ortho-position in the arene moiety produced a lowering of the enantioselection of the corresponding major syn-3 diastereoisomers (Table 2, entries 3-5). However, halogenated aryl groups provided the highest ee in these series (Table 2, entries 4 and 5) with moderate diastereoselection. The $m$-ubstituted chloroarene in imine 2 moiety afforded the best diastereoselection of the whole study but again very low enantiomeric excess was found for both the syn- and anti-isomers $3 \mathbf{e}$ (Table 2, entry 6). The effect of the para-substitution was very similar to those observed in the examples run with ortho-substituted arenes, that means low ee for the examples whose substituents were different from halogenated atoms (Table 2, entries 7-11). In this case 4-fluoro and 4-bromoarenes gave 99\% ee and $80 \%$ ee, of the major syn-diastereoisomers 3h and $\mathbf{3 j}$, respectively. Heterocyclic substituents bonded to the imino group were also good precursors for a high enantioselective Mannich-type addition. Thus, 2-furyl derivative afforded 92\% ee of 3k and 2-thienyl surrogate furnished 78\% ee of $3 \mathbf{l}$ with a 75:25 $d r$ in both cases (Table 2, entries 12-13). Despite being the reaction completed, chemical yields were moderate due to the instability of the final iminoesters $\mathbf{3}$ during purification using flash chromatography. 


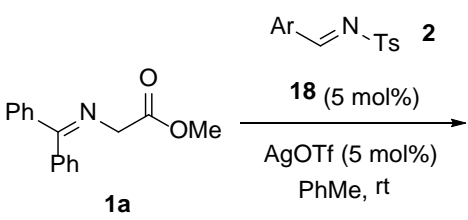

Scheme 3

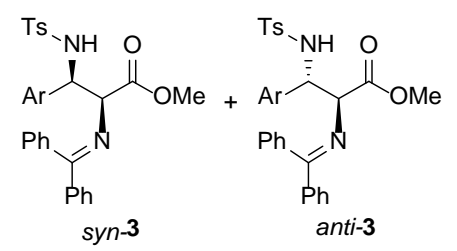

Table 2. Scope of the Mannich-type reaction.

\begin{tabular}{|c|c|c|c|c|c|c|}
\hline Ent. & $\mathrm{Ar}$ & 2 & 3 & Yield (\%) & $d r(\text { syn:anti) })^{\mathrm{a}}$ & $e e(\text { syn, anti) })^{\mathrm{b}}$ \\
\hline 1 & $\mathrm{Ph}$ & $2 a$ & $3 a$ & 70 & $72: 28$ & 96,58 \\
\hline 2 & $\mathrm{Ph}^{\mathrm{c}}$ & $2 a$ & 3a & 70 & $72: 28$ & $-96,-58$ \\
\hline 3 & $2-\mathrm{MeC}_{6} \mathrm{H}_{4}$ & $2 \mathbf{b}$ & $3 b$ & 50 & $80: 20$ & 14,4 \\
\hline 4 & $2-\mathrm{ClC}_{6} \mathrm{H}_{4}$ & 2c & $3 c$ & 55 & $56: 44$ & $46,<5$ \\
\hline 5 & $2-\mathrm{BrC}_{6} \mathrm{H}_{4}$ & 2d & 3d & 67 & $60: 40$ & 48,34 \\
\hline 6 & 3- $\mathrm{ClC}_{6} \mathrm{H}_{4}$ & $2 e$ & $3 \mathbf{e}$ & 42 & $90: 10$ & 14,14 \\
\hline 7 & 4- $\left(\mathrm{CF}_{3}\right) \mathrm{C}_{6} \mathrm{H}_{4}$ & $2 f$ & $3 f$ & 30 & $75: 25$ & 15,2 \\
\hline 8 & 4- $\left(\mathrm{NO}_{2}\right) \mathrm{C}_{6} \mathrm{H}_{4}$ & $2 g$ & $3 g$ & 47 & 70:30 & 20,12 \\
\hline 9 & 4- $\mathrm{FC}_{6} \mathrm{H}_{4}$ & $2 h$ & $3 h$ & 48 & $70: 30$ & 99,10 \\
\hline 10 & $4-\mathrm{ClC}_{6} \mathrm{H}_{4}$ & $2 \mathbf{i}$ & $3 \mathbf{i}$ & 30 & $70: 30$ & 42,16 \\
\hline 11 & 4- $\mathrm{BrC}_{6} \mathrm{H}_{4}$ & $2 \mathbf{j}$ & $3 \mathbf{j}$ & 35 & $50: 50$ & 80,10 \\
\hline 12 & 2-furyl & $2 k$ & $3 k$ & 45 & $75: 25$ & 92,24 \\
\hline 13 & 2-thienyl & 21 & 31 & 45 & 75:25 & 78,38 \\
\hline
\end{tabular}

${ }^{\mathrm{a}}$ Isolated yield after column chromatography.

${ }^{\mathrm{b}}$ Determined by HPLC using chiral columns.

${ }^{\mathrm{c}}$ Reaction performed in the presence of $\operatorname{AgOTf} \cdot\left(R_{\mathrm{a}}, S, S\right)-\mathbf{1 8}$.

This Mannich type reaction can be rationalized on the basis of the models proposed by different authors. $8^{\mathrm{f}}$ The freshly generated azomethine ylide is coordinated by silver atom and the nucleophilic attack occurs to the tosyl imine whose arylsulfonyl group is placed far away from the benzylidene moiety of the dipole (Figure 3). In this transition state an additional coordination between silver and sulfonamido group cannot be ruled out.
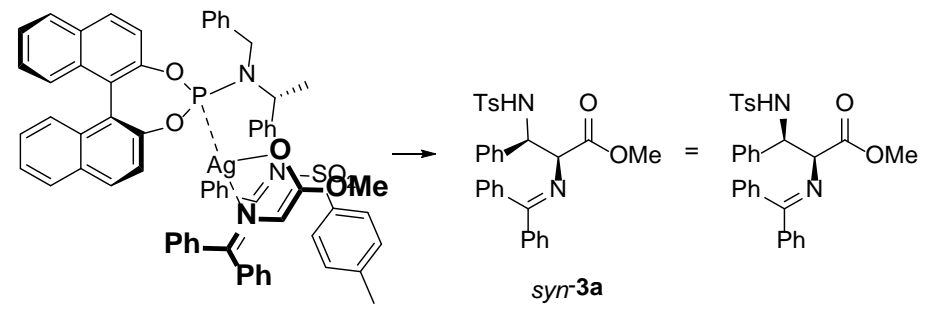

Figure 3. Proposed transition state leading to the syn-stereoisomer. 


\section{Conclusions}

The chiral complex formed by phosphoramidite $\mathbf{1 8}$ and AgOTf (1:1 molar ratio) was the most effective catalyst in the Mannich-type reaction between imino esters derived from benzophenone and $N$-tosylimines. No extra base was needed for the reaction completion, so it acted firstly as Lewis acid coordinating the imino ester and the corresponding counteranion has the base role required for the $\alpha$-deprotonation. Both chemical yields and diastereomeric ratios of the major synproducts were moderate to good. The syn-selectivity was in agreement with the data reported in the literature. The presence of heteroatoms and para-halogenated arenes in the aromatic part of the imine afforded the best enantioselections (up to 99\% ee).

Acknowledgements: This work has been supported by the Spanish Ministerio de Ciencia e Innovación (MICINN) (Consolider INGENIO 2010 CSD2007-00006, CTQ2010-20387, CTQ2013-43446-P), FEDER, Generalitat Valenciana (PROMETEO/2009/039, and PROMETEOII 2014/017), and by the University of Alicante. L. S. thanks University of Edinburgh for an ERASMUS fellowship.

\section{Experimental Part}

General. $N$-Protected imines 2 were prepared according to the published methods.8 Anhydrous solvents were freshly distilled under an argon atmosphere and degasified by Freeze-Pump-Thaw methodology. Aldehydes were also distilled prior to use for the elaboration of the imino esters $\mathbf{1}$ and imines $\mathbf{2}$. Melting points were determined with a Reichert Thermowar hot plate apparatus and are uncorrected. Only the structurally most important peaks of the IR spectra (recorded whit a FT-IR 4100LE (JASCO) (PIKE MIRacle ATR) are listed. ${ }^{1} \mathrm{H}$ NMR (300 MHz) and ${ }^{13} \mathrm{C}$ NMR $(75 \mathrm{MHz})$ spectra were obtained with a Bruker AC-300 by using $\mathrm{CDCl}_{3}$ as solvent and TMS as the internal standard, unless otherwise stated. Optical rotations were measured with a Perkin-Elmer 341polarimeter. HPLC analyses were performed with a JASCO-2000 series equipped with a chiral column (detailed for each compound in the main text) by using mixtures of $n$-hexane/isopropyl alcohol as the mobile phase at $25{ }^{\circ} \mathrm{C}$. Low-resolution electron impact (EI) mass spectra were obtained with a Shimadzu QP-5000 by injection or DIP, and high-resolution mass spectra were obtained with a Finnigan VG Platform or a Finnigan MAT 95S. Analitical TLC was performed on Schleicher \& Schuell F1400/LS 254 silica gel plates and the spots were visualized under UV light $(\lambda=254$ $\mathrm{nm})$. Merck silica gel 60 (0.040-0.063 mm) was used for flash chromatography.

\section{General procedure for the Mannich-type reaction.}

In a $10 \mathrm{ml}$ vial covered by aluminum foil, was added AgOTf (1.54 mg, $0.006 \mathrm{mmol}$ ), phosphoramidite 18 (3.24 mg, $0.006 \mathrm{mmol})$ and toluene $(2 \mathrm{ml})$, and the mixture was stirred at room temperature for $1 \mathrm{~h}$. Benzophenone imine derivative $1(0.120 \mathrm{mmol})$ and $N$-tosylimine $2(0.144 \mathrm{mmol})$ were added. The reaction was stirred $1 \mathrm{~d}$ at room temperature and the crude was analyzed by ${ }^{1} \mathrm{H}$ RMN spectroscopy to determine the diastereomeric ratio, and then purified by flash chromatography (n-hexane:EtOAc; $15 \%$ of EtOAc), affording the products syn/anti-3.

Methyl 2-[(diphenylmethylene)amino]-3-(4-methylphenylsulfonamide)-3-phenylpropanoate syn-3a + anti-3a:8k 42.9 mg; 70\% yield; HPLC: (Daicel Chiralpak AD-H), hexane/i-PrOH 90/10, flow rate $1 \mathrm{ml} / \mathrm{min}$, $\mathrm{t}_{\mathrm{R}}$ : $23.7 \mathrm{~min}(\mathrm{syn}), 36.4 \mathrm{~min}$ (anti), $42.2 \mathrm{~min}$ (anti) and $45 \mathrm{~min}$ (syn), $254 \mathrm{~nm}$; IR $v_{\max }$ : 3297, 1736, 1628, 1328, $1157 \mathrm{~cm}^{-1}$; ${ }^{1} \mathrm{H}-\mathrm{RMN} \delta_{\mathrm{H}}: 2.27\left(\mathrm{~s}, 3 \mathrm{H}, \mathrm{Me}_{\text {anti }}\right), 2.34\left(\mathrm{~s}, 3 \mathrm{H}, \mathrm{Me}_{\text {syn }}\right), 3.49\left(\mathrm{~s}, 3 \mathrm{H}, \mathrm{OMe}_{\text {anti }}\right), 3.51(\mathrm{~s}, 3 \mathrm{H}$, $\left.\mathrm{OMe}_{\text {syn }}\right), 4.14\left(\mathrm{~d}, J=2.2 \mathrm{~Hz}, 1 \mathrm{H}_{\text {syn }}\right), 4.36\left(\mathrm{~d}, J=5.9 \mathrm{~Hz}, 1 \mathrm{H}_{\text {anti }}\right), 4.71\left(\mathrm{dd}, J=7.6,5.8 \mathrm{~Hz}, 1 \mathrm{H}_{\text {anti }}\right), 5.16(\mathrm{dd}, J$ = 8.4, $\left.2.0 \mathrm{~Hz}, 1 \mathrm{H}_{\text {syn }}\right), 5.78\left(\mathrm{~d}, J=7.1 \mathrm{~Hz}, \mathrm{NH}_{\text {anti }}\right), 6.37\left(\mathrm{t}, J=8.4 \mathrm{~Hz}, 2 \mathrm{H}_{\text {syn }}+\mathrm{NH}_{\text {syn }}\right) ; 6.88-7.72\left(\mathrm{~m}, 19 \mathrm{H}_{\text {syn }}\right.$, $\left.19 \mathrm{H}_{\text {anti }}, \operatorname{Ar~H}\right) ;{ }^{13} \mathrm{C}-\mathrm{RMN} \delta_{\mathrm{C}}$ : $21.4(\mathrm{Me}), 52.3\left(\mathrm{C}_{\text {syn }} \mathrm{NH}\right), 59.4\left(\mathrm{C}_{\text {syn }} \mathrm{N}=\right), 60.0\left(\mathrm{C}_{\text {anti }} \mathrm{N}=\right), 69.4\left(\mathrm{OMe}_{\text {anti }}\right), 69.9$ $\left(\mathrm{OMe}_{\text {syn }}\right), 126.8,126.9,127.1,127.3,127.4,128.1,128.3,128.6,128.9,129.2,130.9,135.5,138.2,138.5$, 139.0, 142.8 (ArC), 169.6 syn $173.1_{\text {syn }}$ (CO, CN). MS (EI) m/z (\%): 512 (0.12), 259 (12), 194 (40), 155 (33), 91 (100), 65 (10). 
Methyl 2-[(diphenylmethylene)amino]-3-(4-methylphenylsulfonamide)-3-(o-methylphenyl)propanoate syn-3b + anti-3b:8k 31.6 mg; 50\% yield; HPLC: (Daicel Chiralpak AD-H), hexane/i-PrOH 85/15, flow rate $1 \mathrm{ml} / \mathrm{min}, \mathrm{t}_{\mathrm{R}}$ : $9.7 \mathrm{~min}$ (syn), $14.0 \mathrm{~min}$ (anti), $15.4 \mathrm{~min}$ (anti) and $19.9 \mathrm{~min}$ (syn), $254 \mathrm{~nm}$; IR $v_{\max }$ : 2987, 1725, 1334, 1265, 1159, 1093, 733, $700 \mathrm{~cm}^{-1}$; ${ }^{1} \mathrm{H}-\mathrm{RMN} \delta_{\mathrm{H}}$ : 1.97 (s, 3H, $\mathrm{Me}_{\text {anti }}$ ), 2.04 (s, 3H, $\mathrm{Me}_{\text {syn }}$ ), 2.26 (s, $\left.3 \mathrm{H}, \mathrm{Me}_{\text {anti }}\right), 2.33\left(\mathrm{~s}, 3 \mathrm{H}, \mathrm{Me}_{\text {syn }}\right.$ ), 3.51 (s, 3H, $\left.\mathrm{OMe}_{\text {syn }}\right), 3.53\left(\mathrm{~s}, 3 \mathrm{H}, \mathrm{OMe}_{\text {anti }}\right), 4.06\left(\mathrm{~d}, J=2.1 \mathrm{~Hz}, 1 \mathrm{H}_{\text {syn }}\right), 4.31$ $\left(\mathrm{d}, J=6.5 \mathrm{~Hz}, 1 \mathrm{H}_{\text {anti }}\right), 4.87\left(\mathrm{~d}, J=6.5 \mathrm{~Hz}, 1 \mathrm{H}_{\text {anti }}\right), 5.40$ (dd, $\left.J=8.5,2.0 \mathrm{~Hz}, 1 \mathrm{H}_{\text {syn }}\right), 6.21(\mathrm{~d}, J=7.5 \mathrm{~Hz}$, $2 \mathrm{H}_{\text {syn }}$ ), 6.42 (d, $\left.J=8.4 \mathrm{~Hz}, \mathrm{NH}_{\text {syn }}\right), 6.79-7.89\left(\mathrm{~m}, 16 \mathrm{H}_{\text {syn }}, 16 \mathrm{H}_{\text {anti }}, \mathrm{Ar} H\right) ;{ }^{13} \mathrm{C}-\mathrm{RMN} \delta_{\mathrm{C}}$ : $21.5(\mathrm{Me}), 52.4$ $\left(\mathrm{C}_{\text {syn }} \mathrm{NH}\right), 55.9\left(\mathrm{C}_{\text {syn }} \mathrm{N}=\right), 125.6,126.5,126.8,127.0,128.3,128.5,128.9,129.2,129.7,130.1,132.4$, $169.7_{\text {syn }} ; 172.9_{\text {syn }}(\mathrm{CO}, \mathrm{CN})$; MS (EI) m/z (\%): 526 (0.23), 194 (42), 165 (12), 118 (78), 117 (13), 91 (100), 65 (15).

Methyl 2-[(diphenylmethylene)amino]-3-(4-methylphenylsulfonamide)-3-(o-chlorophenyl)propanoate syn-3c + anti-3c:8 $8^{\mathrm{k}} 36.1 \mathrm{mg} ;$ 55\% yield; HPLC: (Daicel Chiralpak IB), hexane/i-PrOH 95/5, flow rate 1 $\mathrm{ml} / \mathrm{min}, \mathrm{t}_{\mathrm{R}}$ : $17.4 \mathrm{~min}$ (syn) and $21.7 \mathrm{~min}$ (syn), $254 \mathrm{~nm}$; IR $v_{\max }$ : 1739, 1597, 1443, 1332, 1159, 1088, 764,

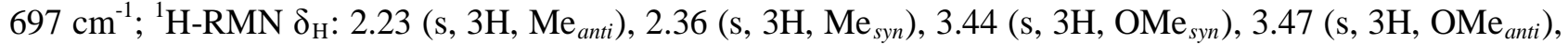
$4.30\left(\mathrm{~d}, J=2.1 \mathrm{~Hz}, 1 \mathrm{H}_{\text {syn }}\right), 4.48\left(\mathrm{~d}, J=5.9 \mathrm{~Hz}, 1 \mathrm{H}_{\text {anti }}\right), 4.85\left(\mathrm{t}, J=5.8 \mathrm{~Hz}, 1 \mathrm{H}_{\text {anti }}\right), 5.54(\mathrm{dd}, J=8.3,2.0 \mathrm{~Hz}$, $1 \mathrm{H}_{\text {syn }}$ ), 6.28 (d, $\left.J=7.3 \mathrm{~Hz}, 2 \mathrm{H}_{\text {syn }}\right), 6.32\left(\mathrm{~d}, J=6.0 \mathrm{~Hz}, \mathrm{NH}_{\text {syn }}\right), 6.46\left(\mathrm{~d}, J=8.3 \mathrm{~Hz}, \mathrm{NH}_{\text {anti }}\right), 6.78-7.73(\mathrm{~m}$, $\left.18 \mathrm{H}_{\text {syn }}, 18 \mathrm{H}_{\text {ant }}, \mathrm{Ar} H\right) ;{ }^{13} \mathrm{C}-\mathrm{RMN} \delta_{\mathrm{C}}$ : $21.4(\mathrm{Me}), 52.0\left(\mathrm{C}_{\text {syn }} \mathrm{NH}\right), 56.2\left(\mathrm{C}_{\text {syn }} \mathrm{N}=\right), 66.6\left(\mathrm{OMe}_{\text {syn }}\right), 126,7,127.1$, 127.5, 127.9, 128.3, 128.6, 129.0, 129.3, 129.7, 130.0, 130.9, 132.4, 135.3, 136.4, 137.8, 138.5, 143.0, 169.9 ${ }_{\text {syn }}, 172.6_{\text {syn }}(\mathrm{CO}, \mathrm{CN})$; MS (EI) m/z (\%): 547 (0.15), 258 (33), 194 (35), 165 (10), 155 (33), 91 (100), $65(10)$.

Methyl 2-[(diphenylmethylene)amino]-3-(4-methylphenylsulfonamide)-3-(o-bromolphenyl)propanoate syn-3d + anti-3d:8k 47.6 mg; 67\% yield; HPLC: (Daicel Chiralpak OD-H), hexane/i-PrOH 90/10, flow rate $0.6 \mathrm{ml} / \mathrm{min}, \mathrm{t}_{\mathrm{R}}$ : $15.2 \mathrm{~min}$ (syn), $16.9 \mathrm{~min}$ (anti), $22.7 \mathrm{~min}$ (anti) and $37.8 \mathrm{~min}$ (syn), $254 \mathrm{~nm}$. IR $v_{\max }$ : 3285, 2987, 1738, 1333, 1159, 1081, 765, $697 \mathrm{~cm}^{-1}$; ${ }^{1} \mathrm{H}-\mathrm{RMN} \delta_{\mathrm{H}}$ : 2.24 (s, 3H, $\mathrm{Me}_{\text {anti }}$ ), 2.36 (s, 3H, Me $\mathrm{syn}_{\text {) }}$ ), 3.45 (s, $\left.3 \mathrm{H}, \mathrm{OMe}_{\text {anti }}\right), 3.46\left(\mathrm{~s}, 3 \mathrm{H}, \mathrm{OMe}_{\text {syn }}\right), 4.33\left(\mathrm{~d}, J=2.1 \mathrm{~Hz}, 1 \mathrm{H}_{\text {syn }}\right), 4.49\left(\mathrm{~d}, J=5.7 \mathrm{~Hz}, 1 \mathrm{H}_{\text {anti }}\right), 4.80(\mathrm{t}, J=5.5$ $\left.\mathrm{Hz}, 1 \mathrm{H}_{\text {anti }}\right), 5.50$ (dd, $\left.J=8.2,2.0 \mathrm{~Hz}, 1 \mathrm{H}_{\text {syn }}\right), 6.26$ (d, $\left.J=6.7 \mathrm{~Hz}, 2 \mathrm{H}_{\text {syn }}\right), 6.39$ (d, $\left.J=8.4 \mathrm{~Hz}, \mathrm{NH}_{\text {anti }}\right), 6.49$ (d, $\left.J=8.2 \mathrm{~Hz}, \mathrm{NH}_{\text {syn }}\right), 6.79-7.87\left(\mathrm{~m}, 18 \mathrm{H}_{\text {syn }}, 18 \mathrm{H}_{\text {anti }}\right.$, Ar $\left.H\right) ;{ }^{13} \mathrm{C}-\mathrm{RMN} \delta_{\mathrm{C}}$ : $21.4(\mathrm{Me}), 51.9\left(\mathrm{C}_{\text {syn }} \mathrm{NH}\right), 58.4$ $\left(\mathrm{C}_{\text {syn }} \mathrm{N}=\right), 66.5\left(\mathrm{OMe}_{\text {syn }}\right), 126.7,127.2,127.3,127.5,127.7,128.0,128.6,129.0,129.3,129.7,130.0,130.9$,

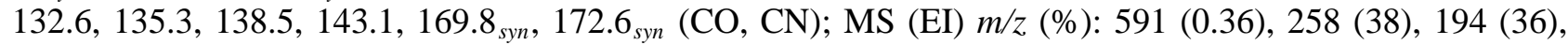
165 (11), 155 (33), 91 (100), 65 (10).

Methyl 2-[(diphenylmethylene)amino]-3-(4-methylphenylsulfonamide)-3-(m-chlorophenyl)propanoate syn-3e + anti-3e:8 27.6 mg; 42\% yield; HPLC: (Daicel Chiralpak AD), hexane/i-PrOH 90/10, flow rate 1 $\mathrm{ml} / \mathrm{min}, \mathrm{t}_{\mathrm{R}}: 40.8 \mathrm{~min}(\mathrm{syn})$ and $78.1 \mathrm{~min}(\mathrm{syn}), 254 \mathrm{~nm}$. IR $v_{\max }$ : 2987, 1739, 1657, 1597, 1159, 1076, 701, $665 \mathrm{~cm}^{-1} ;{ }^{1} \mathrm{H}-\mathrm{RMN} \delta_{\mathrm{H}}: 2.30\left(\mathrm{~s}, 3 \mathrm{H}, \mathrm{Me}_{\text {anti }}\right), 2.36\left(\mathrm{~s}, 3 \mathrm{H}, \mathrm{Me}_{\text {syn }}\right), 3.50\left(\mathrm{~s}, 3 \mathrm{H}, \mathrm{OMe}_{\text {anti }}\right), 3.51\left(\mathrm{~s}, 3 \mathrm{H}, \mathrm{OMe}_{\text {syn }}\right)$, 4.15 (d, $J=2.3 \mathrm{~Hz}, 1 \mathrm{H}_{\text {syn }}$ ), 4.42 (d, $J=5.4 \mathrm{~Hz}, 1 \mathrm{H}_{\text {anti }}$ ), 4.78 (dd, $\left.J=7.7,5.5 \mathrm{~Hz}, 1 \mathrm{H}_{\text {anti }}\right), 5.16$ (dd, $J=8.3$, $\left.2.3 \mathrm{~Hz}, 1 \mathrm{H}_{\text {syn }}\right), 5.77\left(\mathrm{~d}, J=7.7 \mathrm{~Hz}, \mathrm{NH}_{\text {anti }}\right), 6.44\left(\mathrm{t}, J=8.4 \mathrm{~Hz}, 2 \mathrm{H}_{\text {syn }}+\mathrm{NH}_{\text {syn }}\right), 6.89-7.69\left(\mathrm{~m}, 16 \mathrm{H}_{\text {syn }}, 16 \mathrm{H}_{\text {antit }}\right.$, Ar H); ${ }^{13} \mathrm{C}-\mathrm{RMN}\left(75 \mathrm{MHz}, \mathrm{CDCl}_{3}\right) \delta_{\mathrm{C}}$ : $21.4(\mathrm{Me}), 29.7,52.5\left(\mathrm{C}_{\text {syn }} \mathrm{NH}\right), 57.4\left(\mathrm{C}_{\text {syn }} \mathrm{N}=\right)$, $69.5\left(\mathrm{OMe}_{\text {syn }}\right), 123.0$, 126.6, 127.0, 128.3, 128.6, 128.9, 129.4, 129.5, 130.0, 131.2, 132.4, 134.6, 134.7, 143.2, 148.4, 148.6,

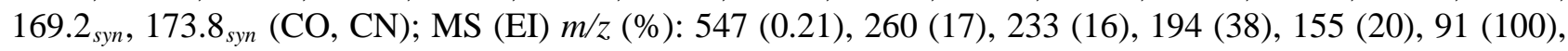
$65(10)$.

Methyl 2-[(diphenylmethylene)amino]-3-(4-methylphenylsulfonamide)-3-(p-trifluoromethylphenyl) propanoate syn-3f + anti-3f: $8^{k} 20.9 \mathrm{mg}$; 30\% yield; HPLC: (Daicel Chiralpak AD-H), hexane/i-PrOH 90/10, flow rate $0.8 \mathrm{ml} / \mathrm{min}, \mathrm{t}_{\mathrm{R}}$ : $13.5 \mathrm{~min}$ (syn), $16.4 \mathrm{~min}$ (anti), $27.8 \mathrm{~min}$ (syn) and $30.5 \mathrm{~min}$ (anti), $254 \mathrm{~nm}$; IR $v_{\max }$ : 3288, 2360, 1743, 1324, 1159, 1119, 1067, $700 \mathrm{~cm}^{-1} ;{ }^{1} \mathrm{H}-\mathrm{RMN} \delta_{\mathrm{H}}: 2.29\left(\mathrm{~s}, 3 \mathrm{H}, \mathrm{Me}_{\text {anti }}\right), 2.36(\mathrm{~s}, 3 \mathrm{H}$, $\mathrm{Me}_{\text {syn }}$ ), 3.52 (s, $\left.3 \mathrm{H}, \mathrm{OMe}_{\text {anti }}\right), 3.54$ (s, 3H, OMe ${ }_{\text {syn }}$ ), 4.17 (d, $\left.J=2.2 \mathrm{~Hz}, 1 \mathrm{H}_{\text {syn }}\right), 4.43\left(\mathrm{~d}, J=5.5 \mathrm{~Hz}, 1 \mathrm{H}_{\text {anti }}\right.$ ), 4.81 (dd, $\left.J=7.6,5.6 \mathrm{~Hz}, 1 \mathrm{H}_{\text {anti }}\right), 5.20$ (dd, $\left.J=8.3,1.9 \mathrm{~Hz}, 1 \mathrm{H}_{\text {syn }}\right), 5.83\left(\mathrm{~d}, J=7.6 \mathrm{~Hz}, \mathrm{NH}_{\text {anti }}\right), 6.39(\mathrm{~d}, J=$ $\left.7.1 \mathrm{~Hz}, 2 \mathrm{H}_{\text {syn }}\right), 6.46$ (d, $\left.J=8.3 \mathrm{~Hz}, \mathrm{NH}_{\text {syn }}\right), 6.87-7.70\left(\mathrm{~m}, 18 \mathrm{H}_{\text {syn }}, 18 \mathrm{H}_{\text {anti }}, \mathrm{Ar} H\right) ;{ }^{13} \mathrm{C}-\mathrm{RMN} \delta_{\mathrm{C}}: 21.3(\mathrm{Me})$, $52.5\left(\mathrm{C}_{\text {syn }} \mathrm{NH}\right), 59.1\left(\mathrm{C}_{\text {syn }} \mathrm{N}=\right), 69.5\left(\mathrm{OMe}_{\text {syn }}\right), 126.7,127.1,127.3,128.2,128.3,128.5,128.8,128.9,129.3$, 130.0, 131.2, 137.8, 138.1, 143.1, 143.2, 169.3 $3_{\text {syn }}, 173.6_{\text {syn }}$ (CO, CN); MS (EI) m/z (\%): 580 (0.25), 252 (10), 194 (35), 165 (10), 155 (46), 91 (100), 65 (10). 
Methyl 2-[(diphenylmethylene)amino]-3-(4-methylphenylsulfonamide)-3-(p-nitrophenyl)propanoate syn-3g + anti-3g:8 31.4 mg; 47\% yield; HPLC: (Daicel Chiralpak OD-H), hexane/i-PrOH 85/15, flow rate $0.6 \mathrm{ml} / \mathrm{min}, \mathrm{t}_{\mathrm{R}}$ : $17.4 \mathrm{~min}$ (syn), $21.0 \mathrm{~min}$ (anti), $23.0 \mathrm{~min}$ (syn) and $39.1 \mathrm{~min}$ (anti), $254 \mathrm{~nm}$; IR $v_{\max }$ : 3273, 1734, 1624, 1519, 1344, 1159, 851, $697 \mathrm{~cm}^{-1}$; ${ }^{1} \mathrm{H}-\mathrm{RMN} \delta_{\mathrm{H}}$ : 2.28 (s, 3H, $\mathrm{Me}_{\text {anti }}$ ), 2.37 (s, 3H, Me $\mathrm{es}_{\text {syn }}$ ), 3.48 (s, $\left.3 \mathrm{H}, \mathrm{OMe}_{\text {syn }}\right), 3.49\left(\mathrm{~s}, 3 \mathrm{H}, \mathrm{OMe}_{\text {anti }}\right), 4.18\left(\mathrm{~d}, J=2.2 \mathrm{~Hz}, 1 \mathrm{H}_{\text {syn }}\right), 4.38\left(\mathrm{~d}, J=5.5 \mathrm{~Hz}, 1 \mathrm{H}_{\text {anti }}\right), 4.78$ (dd, $J=7.5$, $\left.5.6 \mathrm{~Hz}, 1 \mathrm{H}_{\text {anti }}\right), 5.20$ (dd, $\left.J=8.0,2.1 \mathrm{~Hz}, 1 \mathrm{H}_{\text {syn }}\right), 5.91\left(\mathrm{~d}, J=7.0 \mathrm{~Hz}, \mathrm{NH}_{\text {anti }}\right), 6.45\left(\mathrm{~d}, J=7.1 \mathrm{~Hz}, 2 \mathrm{H}_{\text {syn }}\right), 6.51$ $\left(\mathrm{d}, J=8.1 \mathrm{~Hz}, \mathrm{NH}_{\text {syn }}\right), 6.82-8.12\left(\mathrm{~m}, 18 \mathrm{H}_{\text {syn }}, 18 \mathrm{H}_{\text {anti }}, \mathrm{Ar} H\right) ;{ }^{13} \mathrm{C}-\mathrm{RMN} \delta_{\mathrm{C}}: 21.4\left(\mathrm{Me}_{\text {syn }}\right), 25.3\left(\mathrm{Me}_{\text {anti }}\right), 52.3$ $\left(\mathrm{C}_{\text {anti }} \mathrm{NH}\right), 52.5\left(\mathrm{C}_{\text {syn }} \mathrm{NH}\right), 58.9\left(\mathrm{C}_{\text {syn }} \mathrm{N}=\right)$, $59.2\left(\mathrm{C}_{\text {anti }} \mathrm{N}=\right), 64.4\left(\mathrm{OMe}_{\text {anti }}\right), 69.2\left(\mathrm{OMe}_{\text {syn }}\right)$, 123.2, 123.3, 126.6, 127.0, 127.2, 127.8, 128.2, 128.3, 128.6, 128.7, 128.8, 128.9, 129.4, 131.3, 135.1, 137.7, 137.9, 143.4, 145.1,

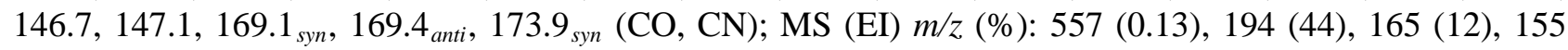
(36), 91 (100), 65 (10).

Methyl 2-[(diphenylmethylene)amino]-3-(4-methylphenylsulfonamide)-3-(p-fluorophenyl)propanoate syn-3h + anti-3h:8 30.6 mg; 48\% yield; HPLC: (Daicel Chiralpak OD-H), hexane/i-PrOH 90/10, flow rate $0.6 \mathrm{ml} / \mathrm{min}, \mathrm{t}_{\mathrm{R}}$ : $13.4 \mathrm{~min}$ (syn), $14.8 \mathrm{~min}$ (anti), $17.1 \mathrm{~min}$ (anti) and $32.6 \mathrm{~min}$ (syn), $254 \mathrm{~nm}$; IR $v_{\max }$ : 3289, 2971, 1753, 1628, 1509, 1428, 1328, 1156, 1081, 736, $697 \mathrm{~cm}^{-1}$; ${ }^{1} \mathrm{H}-\mathrm{RMN} \delta_{\mathrm{H}}$ : 2.29 (s, 3H, $\mathrm{Me}_{\text {anti }}$ ), 2.36 (s, $\left.3 \mathrm{H}, \mathrm{Me}_{\text {syn }}\right), 3.50\left(\mathrm{~s}, 3 \mathrm{H}+3 \mathrm{H}, \mathrm{OMe}_{\text {syn+anti }}\right), 4.12\left(\mathrm{~d}, J=2.3 \mathrm{~Hz}, 1 \mathrm{H}_{\text {syn }}\right), 4.34\left(\mathrm{~d}, J=5.8 \mathrm{~Hz}, 1 \mathrm{H}_{\text {anti }}\right), 4.71$ (dd, $J$ $\left.=7.1,6.1 \mathrm{~Hz}, 1 \mathrm{H}_{\text {ant }}\right), 5.13\left(\mathrm{dd}, J=8.1,2.0 \mathrm{~Hz}, 1 \mathrm{H}_{\text {syn }}\right), 5.80\left(\mathrm{~d}, J=7.5 \mathrm{~Hz}, \mathrm{NH}_{\text {anti }}\right), 6.42\left(\mathrm{t}, J=7.5 \mathrm{~Hz}, 2 \mathrm{H}_{\text {syn }}\right.$ $\left.+\mathrm{NH}_{\text {syn }}\right), 6.74-7.90\left(\mathrm{~m}, 18 \mathrm{H}_{\text {syn }}, 18 \mathrm{H}_{\text {anti }}, \mathrm{Ar} \mathrm{H}\right) ;{ }^{13} \mathrm{C}-\mathrm{RMN} \delta_{\mathrm{C}}: 21.4(\mathrm{Me}), 52.4\left(\mathrm{C}_{\text {syn }} \mathrm{NH}\right), 58.8\left(\mathrm{C}_{\text {syn }} \mathrm{N}=\right), 69.9$ $\left(\mathrm{OMe}_{\text {syn }}\right), 114.9,126.5,126.8,127.0,128.2,128.4,128.9,129.2,129.7,130.0,131.0,132.4,138.0,142.9$, 169.5 syn, 173.4 syn $(\mathrm{CO}, \mathrm{CN})$; MS (EI) m/z (\%): 530 (0.32), 277 (10), 194 (37), 155 (35), 91 (100), 65 (10).

Methyl 2-[(diphenylmethylene)amino]-3-(4-methylphenylsulfonamide)-3-(p-chlorophenyl)propanoate syn-3i + anti-i:8k 46.2 mg; 70\% yield; HPLC: (Daicel Chiralpak OD-H), hexane/i-PrOH 90/10, flow rate 0.6 $\mathrm{ml} / \mathrm{min}, \mathrm{t}_{\mathrm{R}}$ : $16.3 \mathrm{~min}$ (syn), $17.6 \mathrm{~min}$ (anti), $21.1 \mathrm{~min}$ (syn) and $37.4 \mathrm{~min}$ (anti), $254 \mathrm{~nm}$; IR $v_{\max }$ : 3260, 2923, 1754, 1597, 1288, 1157, 1088, 698, $662 \mathrm{~cm}^{-1}$; ${ }^{1} \mathrm{H}-\mathrm{RMN} \delta_{\mathrm{H}}: 2.30$ (s, 3H, $\mathrm{Me}_{\text {anti }}$ ), 2.37 (s, 3H, $\mathrm{Me}_{\text {syn }}$ ), 3.50 (s, $\left.3 \mathrm{H}+3 \mathrm{H}, \mathrm{OMe}_{\text {syn+anti }}\right), 4.13\left(\mathrm{~d}, J=2.3 \mathrm{~Hz}, 1 \mathrm{H}_{\text {syn }}\right), 4.35\left(\mathrm{~d}, J=5.7 \mathrm{~Hz}, 1 \mathrm{H}_{\text {anti }}\right), 4.69(\mathrm{dd}, J=7.3,5.8 \mathrm{~Hz}$, $\left.1 \mathrm{H}_{\text {anti }}\right), 5.11\left(\mathrm{dd}, J=8.3,2.1 \mathrm{~Hz}, 1 \mathrm{H}_{\text {syn }}\right), 5.79\left(\mathrm{~d}, J=7.6 \mathrm{~Hz}, \mathrm{NH}_{\text {anti }}\right), 6.41\left(\mathrm{~d}, J=9.1 \mathrm{~Hz}, \mathrm{NH}_{\text {syn }}\right), 6.44(\mathrm{~d}, J=$ $\left.7.4 \mathrm{~Hz}, 2 \mathrm{H}_{\text {syn }}\right), 6.93-7.88\left(\mathrm{~m}, 18 \mathrm{H}_{\text {syn }}, 18 \mathrm{H}_{\text {anti }}, \mathrm{Ar} \mathrm{H}\right) ;{ }^{13} \mathrm{C}-\mathrm{RMN} \delta_{\mathrm{C}}$ : $21.4(\mathrm{Me}), 52.4\left(\mathrm{C}_{\text {syn }} \mathrm{NH}\right), 58.9\left(\mathrm{C}_{\text {syn }} \mathrm{N}=\right)$, $69.7\left(\mathrm{OMe}_{\text {syn }}\right), 126.4,126.8,127.0,128.2,128.5,128.9,129.3,129.7,130.0,131.1,133.2,135.3,137.5$, 138.2, 143.1, 169.5 syn, 173.5 syn $(\mathrm{CO}, \mathrm{CN})$; MS (EI) m/z (\%): 547 (0.18), 194 (36), 155 (40), 91 (100), 65 (10).

Methyl 2-[(diphenylmethylene)amino]-3-(4-methylphenylsulfonamide)-3-(p-bromophenyl)propanoate syn-3j + anti-3j:8h 24.8 mg; 35\% yield; HPLC: (Daicel Chiralpak OD-H), hexane/i-PrOH 90/10, flow rate $0.6 \mathrm{ml} / \mathrm{min}, \mathrm{t}_{\mathrm{R}}: 13.3 \mathrm{~min}$ (syn), $14.7 \mathrm{~min}$ (anti), $16.8 \mathrm{~min}$ (syn) and $29.7 \mathrm{~min}$ (anti), $254 \mathrm{~nm}$; IR $v_{\text {max }}$ : 3296, 2971, 1754, 1630, 1328, 1157, 1075, $698 \mathrm{~cm}^{-1} ;{ }^{1} \mathrm{H}-\mathrm{RMN} \delta_{\mathrm{H}}$ : 2.31 (s, 3H, $\mathrm{Me}_{\text {anti }}$ ), 2.37 (s, 3H, Me $\mathrm{e}_{\text {syn }}$ ), 3.50 (s, $\left.3 \mathrm{H}+3 \mathrm{H}, \mathrm{OMe}_{\text {syn+anti }}\right), 4.13\left(\mathrm{~d}, J=2.3 \mathrm{~Hz}, 1 \mathrm{H}_{\text {syn }}\right), 4.35\left(\mathrm{~d}, J=5.6 \mathrm{~Hz}, 1 \mathrm{H}_{\text {anti }}\right), 4.67(\mathrm{dd}, J=7.5,5.7 \mathrm{~Hz}$, $\left.1 \mathrm{H}_{\text {anti }}\right), 5.08$ (dd, $\left.J=8.3,2.1 \mathrm{~Hz}, 1 \mathrm{H}_{\text {syn }}\right), 5.74\left(\mathrm{~d}, \mathrm{~J}=7.4 \mathrm{~Hz}, \mathrm{NH}_{\text {anti }}\right), 6.37$ (d, $\left.J=8.3 \mathrm{~Hz}, \mathrm{NH}_{\text {syn }}\right), 6.45(\mathrm{~d}, J=$ $\left.7.1 \mathrm{~Hz}, 2 \mathrm{H}_{\text {syn }}\right), 6.85-7.69\left(\mathrm{~m}, 18 \mathrm{H}_{\text {syn }}, 18 \mathrm{H}_{\text {anti }}, \mathrm{Ar} H\right)$; ${ }^{13} \mathrm{C}-\mathrm{RMN} \delta_{\mathrm{C}}$ : $21.4(\mathrm{Me}), 29.7,52.4\left(\mathrm{C}_{\text {syn }} \mathrm{NH}\right), 58.9$ $\left(\mathrm{C}_{\text {syn }} \mathrm{N}=\right)$, $69.6\left(\mathrm{OMe}_{\text {syn }}\right), 126.8,127.1,128.2,128.5,128.6,128.9,129.3,130.0,131.2,138.2,143.1$,

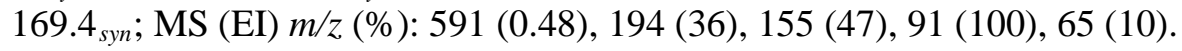

Methyl 2-[(diphenylmethylene)amino]-3-(4-methylphenylsulfonamide)-3-(2-furyl)propanoate syn-3k + anti-3k:8 ${ }^{\mathrm{d}} 27.1 \mathrm{mg}$; 45\% yield; HPLC: (Daicel Chiralpak AD), hexane/i-PrOH 90/10, flow rate $1 \mathrm{ml} / \mathrm{min}, \mathrm{t}_{\mathrm{R}}$ : $15.0 \mathrm{~min}$ (syn), $25.1 \mathrm{~min}$ (anti), $40.5 \mathrm{~min}$ (anti) and $65.5 \mathrm{~min}$ (syn), $254 \mathrm{~nm}$; IR $v_{\max }$ : 3267, 2971, 2360, 1739, 1277, 1158, 1075, 812, 700, $665 \mathrm{~cm}^{-1} ;{ }^{1} \mathrm{H}-\mathrm{RMN} \delta_{\mathrm{H}}: 2.37$ (s, 3H, $\mathrm{Me}_{\text {anti }}$ ), 2.37 (s, 3H, $\mathrm{Me}_{\text {syn }}$ ), $3.52(\mathrm{~s}$, $\left.3 \mathrm{H}, \mathrm{OMe}_{\text {anti }}\right), 3.58\left(\mathrm{~s}, 3 \mathrm{H}, \mathrm{OMe}_{\text {syn }}\right), 4.26\left(\mathrm{~d}, J=2.3 \mathrm{~Hz}, 1 \mathrm{H}_{\text {syn }}\right), 4.58\left(\mathrm{~d}, J=4.3 \mathrm{~Hz}, 1 \mathrm{H}_{\text {anti }}\right), 5.24(\mathrm{dd}, J=9.1$, $4.2 \mathrm{~Hz}, 1 \mathrm{H}_{\text {anti }}$ ), 5.45 (dd, $\left.J=8.8,1.9 \mathrm{~Hz}, 1 \mathrm{H}_{\text {syn }}\right), 6.35$ (d, $\left.J=9.1 \mathrm{~Hz}, \mathrm{NH}_{\text {anti }}\right), 6.75\left(\mathrm{~m}, 4 \mathrm{H}_{\text {syn }}+\mathrm{NH}_{\text {syn }}\right.$ ), 7.03$7.94\left(\mathrm{~m}, 14 \mathrm{H}_{\text {syn }}, 14 \mathrm{H}_{\text {anti }}\right.$, Ar H); ${ }^{13} \mathrm{C}-\mathrm{RMN} \delta_{\mathrm{C}}: 21.4(\mathrm{Me}), 52.5\left(\mathrm{C}_{\text {syn }} \mathrm{NH}\right), 55.9\left(\mathrm{C}_{\text {syn }} \mathrm{N}=\right), 69.9\left(\mathrm{OMe}_{\text {syn }}\right)$, 125.3, 126.2, 127.0, 128.2, 128.3, 128.5, 128.8, 129.1, 129.2, 130.1, 131.0, 132.4, 142.8; MS (EI) $\mathrm{m} / \mathrm{z}(\%)$ : 502 (0.56), 194 (40), 155 (23), 92 (11), 91 (100), 65 (10).

Methyl 2-[(diphenylmethylene)amino]-3-(4-methylphenylsulfonamide)-3-(2-thienyl)propanoate syn-31

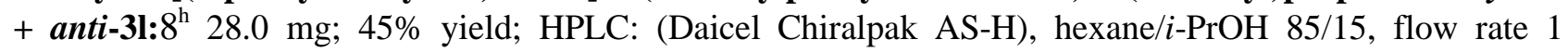


$\mathrm{ml} / \mathrm{min}, \mathrm{t}_{\mathrm{R}}$ : $15.3 \min (s y n)$ and $22.7 \mathrm{~min}(s y n), 254 \mathrm{~nm}$; IR $v_{\max }$ : 3269, 2357, 1742, 1572, 1329, 1156, 1089, 812, 734, $700 \mathrm{~cm}^{-1}$; ${ }^{1} \mathrm{H}-\mathrm{RMN} \delta_{\mathrm{H}}: 2.34$ (s, 3H, Me anti), $2.38\left(\mathrm{~s}, 3 \mathrm{H}, \mathrm{Me}_{\text {syn }}\right.$ ), $3.51\left(\mathrm{~s}, 3 \mathrm{H}+3 \mathrm{H}, \mathrm{OMe}_{\text {syn+anti }}\right.$ ), $4.37\left(\mathrm{~d}, J=2.5 \mathrm{~Hz}, 1 \mathrm{H}_{\text {syn }}\right), 4.47\left(\mathrm{~d}, J=6.0 \mathrm{~Hz}, 1 \mathrm{H}_{\text {anti }}\right), 5.20$ (dd, $\left.J=9.3,2.0 \mathrm{~Hz}, 1 \mathrm{H}_{\text {syn }}\right), 6.07$ (dd, $J=2.5,0.8$ $\left.\mathrm{Hz}, 1 \mathrm{H}_{\text {syn }}\right), 6.15$ (dd, $\left.J=3.2,1.9 \mathrm{~Hz}, 1 \mathrm{H}_{\text {syn }}\right), 6.22\left(\mathrm{~d}, J=9.3 \mathrm{~Hz}, 1 \mathrm{H}_{\text {syn }}\right), 6.75\left(\mathrm{~d}, J=1.6 \mathrm{~Hz}, 1 \mathrm{H}_{\text {syn }}\right), 6.77$ (d, $J$ $\left.=1.2 \mathrm{~Hz}, \mathrm{NH}_{\text {syn }}\right), 7.08-7.89\left(\mathrm{~m}, 14 \mathrm{H}_{\text {syn }}, 14 \mathrm{H}_{\text {anti }}, \operatorname{Ar~H}\right) ;{ }^{13} \mathrm{C}-\mathrm{RMN} \delta_{\mathrm{C}}: 21.4(\mathrm{Me}), 52.5\left(\mathrm{C}_{\text {syn }} \mathrm{NH}\right), 55.9$ $\left(\mathrm{C}_{\text {syn }} \mathrm{N}=\right), 69.9\left(\mathrm{OMe}_{\text {syn }}\right), 107.8,110.4,125.3,125.5,126.2,126.4,127.0,127.9,128.1,128.5,128.8,129.0$, 129.2, 129.3, 129.7, 131.0, 136.7, 139.0, 162.2, 169.3 $3_{\text {syn }}, 173.8_{\text {syn }}(\mathrm{CO}, \mathrm{CN})$; MS (EI) $\mathrm{m} / \mathrm{z}(\%): 518$ (0.47), 194 (40), 165 (10), 155 (22), 91 (100), 65 (10).

\section{References}

${ }^{1}$ Subramaniapillai, S. G. J. Chem. Sci. 2013, 125, 467-482.

2 Bhadury, P. S.; Yang, S.; Song, B.-A. Curr. Org. Synth. 2012, 9, 695-726; b) Kiss, L.; Fulop, F. Chem. Rev. 2014, 114, 1116-1169.

${ }^{3}$ For recent general reviews, see: a) Karimi, B.; Enders, D.; Jafari, E. Synthesis 2013, 45, 2769-2812; b) Córdova, A. in Stereoselective Organocatalysis. Ed. Ríos-Torres, R. Wiley, Weinheim, 2013; c) Gómez-Arrayás, R.; Carretero, J. C. Chem. Soc. Rev. 2009, 38, 1940-1948.

${ }^{4}$ a) This structural arrangement is very common in nature: Viso, A.; Fernández de la Pradilla, R.; García, A.; Flores, A. Chem Rev. 2005, 105, 3167-3196; b) This strategy has been used for the synthesis of antibacterial agents: Patterson, B. D.; Lu, Q.; Aggen, J. B.; Dozzo, P.; Kasar, R. A.; Linsell, M. S.; Kane, Timothy, R.; Gliedt, M. J.; Hildebrandt, D. J.; McEnroe, G. A. PCT Int. Appl. (2013), WO 2013170030 A1 20131114. CAN: 159:731375.

${ }^{5}$ For a recent example, see: Callebaut, G.; Mangelinckx, S.; Kiss, L.; Sillanpää, R.; Fülöp F.; De Kimpe, N. Org. Biomol. Chem. 2012, 10, 2326-2338.

${ }^{6}$ Wang, J.; Zhang, L.; Jiang, H.; Liu, H. Curr. Pharm. Des. 2010, 16, 1252-1259.

7 a) Okada, A.; Shibuguchi, T.; Ohshima, T.; Masu, H.; Yamaguchi, K.; Shibasaki, M. Angew. Chem. Int. Ed. 2005, 44, 4564-4567; b) Bandar, J.; Lambert, T. H. J. Am. Chem. Soc. 2013, 135, 11799-11802.

8 a) Juhl, K.; Gathergood, N.; Jorgensen, K. A. Angew. Chem. Int. Ed. 2001, 40, 2995-2997; b) Bernardi, L.; Gothelf, A. S.; Hazell, R. G.; Jorgensen, K. A. J. Org. Chem. 2003, 68, 2583-2591; c) Hernández-Toribio, J.; Gómez-Arrayás, R.; Carretero, J. C. J. Am. Chem. Soc. 2008, 130, 16150-16151; d) Yan, X.-X.; Peng, Q.; Li, Q.; Zhang, K.; Yao, J.; Hou, X.-L.; Wu, Y.-D. J. Am. Chem. Soc. 2008, 130, 14362-14363; e) Shang, D.; Liu, Y.; Zhou, X.; Liu, X.; Feng, X. Chem. Eur. J. 2009, 15, 3678-3681; f) Hernández-Toribio, J.; Gómez-Arrayás, R.; Carretero, J. C. Chem. Eur. J. 2010, 16, 1153-1157; g) Liang, G.; Tong, M.-C.; Tao, H.; Wang, C.-J. Adv. Synth. Catal. 2010, 352, 1851-1855; h) Arai, T.; Mishiro, A.; Matsumura, E.; Awata, A.; Shirasugi, M. Chem. Eur. J. 2012, 18, 11219-11222; i) Hernando, E.; GómezArrayás, R.; Carretero, J. C. Chem. Commun. 2012, 48, 9622-9624; j) Yamashita, Y.; Yoshimoto, S.; Masuda, K.; Kobayashi, S. Asian J. Chem. 2012, 1, 327-330; k) Imae, K. Shimizu, K.; Ogata, K.; Fukuzawa, S.-I. J. Org. Chem. 2011, 76, 3604-3608. Yamashita, Y.; Yoshimoto, S.; Masuda, K.; Kobayashi, S. Asian J. Chem. 2012, 1, $327-330$.

${ }^{9}$ Imino nitriles have been also tested as dipole precursors: Yamashita, Y.; Matsumoto, M.; Chen, Y.-J.; Kobayashi, S. Tetrahedron 2012, 68, 7558-7563.

10 a) Teichert, J. F.; Feringa, B. L. Angew. Chem. Int. Ed. 2010, 49, 2486-2528; b) Privileged Chiral Ligands and Catalysts; Qi-Lin Zhou, Ed.; Wiley-VCH: New York, 2011.

${ }^{11}$ a) Martín-Rodríguez, M.; Nájera, C.; Sansano, J. M.; Costa, P. R. R.; Crizanto de Lima, E.; Dias, A. G. Synlett 2010, 962-966; b) Martín-Rodríguez, M.; Nájera, C.; Sansano, J. M.; de Cózar, A.; Cossío, F. P. Chem. Eur. J. 2011, 17, 14224-14233; c) Mancebo-Aracil, J.; Martín-Rodríguez, M.; Nájera, C.; Sansano, J. M.; Costa, P. R. R.; Crizanto de Lima, E.; Dias, A. G. Tetrahedron: Asymmetry 2012, 23, 1596-1606.

12 a) Nájera, C.; Retamosa, M. G.; Sansano, J. M. Angew. Chem. Int. Ed. 2008, 47, 6055-6058; b) Nájera, C.; Retamosa, M. G.; Martín-Rodríguez, M.; Sansano, J. M.; de Cózar, A.; Cossío, F. P. Eur. J. Org. Chem. 2009, 56225634; c) Castelló, L. M.; Nájera, C.; Sansano, J. M.; Larrañaga, O.; de Cózar, A.; Cossío, F. P. Org. Lett. 2013, 15, 2902-2905.

13 a) Synthetic Applications of 1,3-Dipolar Cycloaddition Chemistry Toward Heterocycles and Natural Products, Padwa, A.; Pearson, W. H. Eds., John Wiley \& Sons: New Jersey, 2003; b) Nájera, C.; Sansano, J. M. Curr. Org. Chem. 2003, 7, 1105-1150; c) Eberbach, W. Sci. Synth. 2004, 27, chp. 11, 441-498; d) Coldham, I.; Hufton, R. Chem. Rev. 2005, 105, 2765-2810; e) Nair, V.; Suja, T. D. Tetrahedron 2007, 63, 12247-12275; Padwa, A.; Bur, S. K. Tetrahedron 2007, 63, 5341-5378; f) Hashimoto, T.; Maruoka, K. Handbook of Cyclization Reactions, Ma, S. Ed. Wiley-VCH: Weinheim 2010.

${ }^{14}$ For more specific recent reviews of asymmetric 1,3-DC, see: a) Pellissier, H. Tetrahedron 2007, 63, 3235-3285; b) Nájera, C.; Sansano J. M. Topics in Heterocyclic Chemistry, A. Hassner, Ed.; Springer-Verlag: Berlin-Heidelberg, 2008, vol. 12, pp. 117-145; Stanley, L. M.; Sibi, M. P. Chem.Rev. 2008, 108, 2887-2902; c) Álvarez-Corral, M.; Muñoz-Dorado, M.; Rodríguez-García, I. Chem. Rev. 2008, 108, 3174-3198; d) Naodovic, M.; Yamamoto, H. Chem. Rev. 2008, 108, 3132-3148; d) Nájera, C.; Sansano, J. M.; Yus, M. J. Braz. Chem. Soc. 2010, 21, 377-412; e) Kissane, 
M.; Maguire, A. R. Chem. Soc. Rev. 2010, 39, 845-883; f) Adrio, J.; Carretero, J. C. Chem. Commun. 2011, 47, 67846794; g) Nájera, C.; Sansano, J. M. J. Organomet. Chem. 2014, 771, 78-92.

${ }^{15}$ Recent references using chiral copper complexes: a) Pascual-Escudero, A.; González-Esguevillas, M.; Padilla, S.; Adrio, J.; Carretero, J. C. Org. Lett. 2014, 16, 2228-2231; b) Tong, M.-C.; Chen, X.; Tao, H. Y.; Wang, C.-J. Angew. Chem. Int. Ed. 2014, 39, 12377-12380; c) Tao, H. Y.; He, Z.-H.; Yang, Y.; Wang, C. J. RSC Adv. 2014, 4, 1689916905; d) Castelló, L. M.; Nájera, C.; Sansano, J. M., Larrañaga, O.; de Cózar, A.; Cossío, F. P. Org Lett. 2013, 15, 2902-2905; e) Li, Q.-H.; Liu, T.-L.; Wei, L.; Zhou, X.; Tao, H.-Y.; Wang, R. Chem. Commun. 2013, 49, 9642-9644; f) Narayan, R.; Bauer, J. O.; Strohmann, C.; Antonchick, A. P.; Waldmann, H. Angew. Chem. Int. Ed. 2013, 38, 12892-12896. Recent references using chiral silver complexes: a) Watanabe, S.; Tada, A.; Tokoro, Y.; Fukuzawa, S.-I. Tetrahedron Lett. 2014, 55, 1306-1309; b) González-Esguevillas, M.; Adrio, J.; Carretero, J. C. Chem. Commun. 2013, 49, 4649-4651; c) Yamashita, Y.; Kobayashi, S. Chem. Eur. J. 2013, 19, 9420-9427; d) Imae, K.; Konno, T.; Ogata, K.; Fukuzawa, S.-I. Org. Lett. 2012, 14, 4410-4413. Recent references using chiral gold complexes: Martín-Rodríguez, M.; Nájera, C.; Sansano, J. M.; de Cózar, A.; Cossío, F. P. Chem. Eur. J. 2011, 17, 14224-14233.

${ }^{16}$ For recent examples of organotalyzed processes, see: a) Wang, L.; Shi, X.-M.; Dong, W.-P.; Zhu, L.-P.; Wang, R. Chem. Commun. 2013, 49, 3458-3460; b) Guo, C.; Song, J.; Gong, L.-Z. Org. Lett. 2013, 15, 2676-2679; c) Shi, F.; Xing, G.-J.; Tan, R.; Zhu, R.-Y.; Tu, S. Org. Biomol. Chem. 2013, 11, 1482-1489; d) Tian, L.; Hu, X.-Q. Li, Y.-H.; Xu, P.-F. Chem. Commun. 2013, 49, 7213-7215; e) Reboredo, S.; Reyes, E.; Vicario, J. L.; Badía, D.; Carrillo, L.; de Cózar, A.; Cossío, F. P. Chem. Eur. J. 2012, 18, 7179-7188.

${ }^{17}$ Chen, Q.-A. Zeng, W.; Wang, D.-W.; Zhou, Y.-G. Synlett 2009, 2236-2241. 